\title{
Oil Content and Fatty Acid Composition of the Seeds of 16 Avocado (Persea americana) Accessions Collected From Southern China and Their Application in a Soap Bar
}

\author{
$\mathrm{Yu} \mathrm{Ge}{ }^{1}$, Xiongyuan $\mathrm{Si}^{2}$, Bin $\mathrm{Wu}^{1}$, Xiangshu Dong ${ }^{3}$, Zining $\mathrm{Xu}^{1} \&$ Weihong $\mathrm{Ma}^{1}$ \\ ${ }^{1}$ Haikou Experimental Station, Chinese Academy of Tropical Agricultural Sciences, Hainan, People's Republic of \\ China \\ ${ }^{2}$ Biotechnology Center, Anhui Agricultural University, Anhui, People’s Republic of China \\ ${ }^{3}$ College of Agriculture, Yunnan University, Yunnan, People's Republic of China \\ Correspondence: Yu Ge, Haikou Experimental Station, Chinese Academy of Tropical Agricultural Sciences, \\ Hainan, People's Republic of China. Tel: 86-089-866-770-005. E-mail: geyu@catas.cn \\ Weihong Ma, Haikou Experimental Station, Chinese Academy of Tropical Agricultural Sciences, Hainan, \\ People's Republic of China. Tel: 86-089-866-773-067. E-mail: zjwhma@163.com
}

\author{
Received: July 18, $2018 \quad$ Accepted: August 24, $2018 \quad$ Online Published: October 15, 2018 \\ doi:10.5539/jas.v10n11p69 URL: https://doi.org/10.5539/jas.v10n11p69
}

The research is financed by the National Natural Science Foundation of China (Grant No. 31701883) and the Central Public-interest Scientific Institution Basal Research Fund for Chinese Academy of Tropical Agricultural Sciences (Grant No. 1630092018003).

\begin{abstract}
The avocado (Persea americana), an edible fruit, is one of the main agricultural products in many tropical regions. Avocado fruit is rich in fat, and commercialized for fresh consumption and industrially processed leaving seed as a major residue. Avocado seed from the industry is worthy of attention for certain industrial applications and feasibility. Transforming avocado seed lipids into ecologically friendly or sustainable materials suitable for the cosmetic industry is promising from the perspective of green and environmental protection. The oil contents and fatty acid compositions of the seeds of 16 avocado accessions collected from southern China were investigated, revealing significant differences among most of the accessions. Seventeen fatty acids were identified and quantified by gas chromatography-mass spectrometry in the seeds of all 16 avocado accessions. Linoleic (40.14\%), palmitic (23.54\%), and oleic acids $(16.23 \%)$ were the major fatty acids in the seeds, and the total contents of unsaturated fatty acids in the seeds were all higher than those of saturated fatty acids. The biochemical properties of the avocado seed oils relevant to their application in industrial practice were examined [e.g., the acid (3.74 $\mathrm{mg} \mathrm{KOH} / \mathrm{g}$ oil), iodine (124.09 $\mathrm{g} \mathrm{I}_{2} / 100 \mathrm{~g}$ oil), peroxide (49.83 meq $\mathrm{H}_{2} \mathrm{O}_{2}$ ), and saponification (167.98 $\mathrm{mg} \mathrm{KOH} / \mathrm{g}$ oil) values]. Furthermore, the bar soap containing avocado seed oil was made, and its physicochemical properties ( $\mathrm{pH}$ and foamability) were evaluated.
\end{abstract}

Keywords: avocado, fatty acids, seed bio-oil, cosmetics

\section{Introduction}

Avocado (Persea americana Mill.), a member of the family Lauraceae of the order Laurales, is a plant native to Central America, South America, and Mexico (Schaffer et al., 2012). The avocado is among the most economically important subtropical/tropical fruit crops in the world, and production and consumption levels having increased dramatically during the last 150 years (Schaffer et al., 2012). In the world, the total area of avocado cultivation has reached 563,916 hectares, with almost ten tons per hectare in the annual production of avocado in 2016 (FAOSTAT, 2018). The consumption of avocado in the whole world has rapidly increased from $3,426,294$ tons in 2008 to $5,567,044$ tons in 2016 (FAOSTAT, 2018). One factor contributing to this marked increase was the constant expansion of avocado products into new markets in parts of the world where avocado was previously unknown or scarcely available (Schaffer et al., 2012). In China, the total area of avocado cultivation has reached over 20,000 hectares, with over six tons per hectare in the annual production of avocado 
in 2016 (FAOSTAT, 2018). The consumption of avocado in China has slowly increased from over 95,000 tons in 2008 to 122,942 tons in 2016 (FAOSTAT, 2018). Nowadays, there are many avocado-planting regions in provinces across southern China cultivating foreign cultivars with superior quality (e.g., Fuerte, Hass, and Shepard) along with native Chinese cultivars and accessions (Ge et al., 2017a).

In recent years, there has been growing interest in identifying phytochemical alternatives to the synthetic substances commonly used in the food, pharmaceutical, and cosmetic industries. This effort was supported by consumer concerns over the safety of products containing synthetic chemicals, which were believed to cause or enhance negative health effects and pollute the environment (Vinha et al., 2013; Galvão et al., 2014). Although vegetable oils have traditionally been used primarily in the food industry, they are gaining applications in, for example, the cosmetic industry (Lourith et al., 2014, 2016). In terms of oil content in the fruit, avocado fruit was exceeded only by the fruits of the palm and olive trees (Knothe, 2013). Remarkably, the lipid content in avocado could reach $5 \%$ to $30 \%$ of the fruit fresh weight, depending on the cultivar, seasonality, and planting conditions (Rodríguez-Carpena et al., 2011; Vinha et al., 2013; Galvão et al., 2014). Avocado fruit lipids contained 50\% to $60 \%$ monounsaturated fatty acids and $10 \%$ to $15 \%$ polyunsaturated linoleic and linolenic acids, which were beneficial to human cardiovascular health (Dreher \& Davenport, 2013). Furthermore, avocado fruit lipids could be used in non-food industries, for example, as an alternative biodiesel source instead of the conventional petroleum-based diesel fuel (Giraldoa \& Moreno-Piraján, 2012; Knothe, 2013). In addition, the high non-saturated content of avocado fruit lipids provided superior skin permeability and sunscreen performance, and could be used in sunscreen cream as the emulsifier (Santo et al., 2014).

Given the increasing demand for vegetable oils in the cosmetic industry (Lourith et al., 2014, 2016; Wu et al., 2015), transforming avocado seed lipids into ecologically friendly or sustainable materials suitable for the cosmetic industry, in particular for personal health products, is promising. In this context, we firstly evaluated and compared the yields and fatty acid compositions of oils from avocado seed. To explore the potential values of the major residue (seed) of avocado fruit, we then determined the physicochemical properties of avocado seed oil to evaluate the oil's applicability in cosmetic applications. In addition, a soap containing avocado seed oil was made and the physicochemical properties of the soap were assessed with respect to possible cosmetic applications.

\section{Materials and Methods}

\subsection{Plant Material, Reagents, and Sample Preparation}

The 16 avocado accessions used in the present study were obtained from Ge et al. (2018). Six ten-year old trees were collected from each accession. Two homogeneous trees were used as a unit with three biological repetitions for each accession. The samples of 18 mature fruits of each biological repetition in each accession were randomly collected when the mesocarp dry matter was $\geq 22 \%$ based on the criterion according to Medina-Carrillo et al. (2017), and immediately transported to the laboratory in standard foam boxes used for export packaging. The fruit samples were maintained at $5{ }^{\circ} \mathrm{C}$ to $6{ }^{\circ} \mathrm{C}$. The seeds were separated from the fruits, homogenized using a domestic blender, and then stored at $4{ }^{\circ} \mathrm{C}$ for a maximum of one week before analysis.

\subsection{Determination of Total Lipid Contents}

Oil content was evaluated using the method of Ge et al. (2017b). Eighteen seeds of each biological repetition in each accession were mixed. The total lipid content was expressed as $\mathrm{g} / 100 \mathrm{~g}$ on a fresh weight (FW) basis. The experiments were performed in three biological replicates for each accession.

\subsection{Gas Chromatography-Mass Spectrometry (GC-MS) Analysis and Fatty Acid Identification}

The fatty acid profiles were determined as described by Ge et al. (2017b). Eighteen seeds of each biological repetition in each accession were mixed. The analyses were performed using an Agilent7890B-7000B GC-MS (Santa Clara, CA, USA) equipped with a DB-5MS column ( $60 \mathrm{~m} \times 0.25 \mathrm{~mm}$ i.d., $0.25-\mu \mathrm{m}$ film thickness) using helium $(1.2 \mathrm{~mL} / \mathrm{min})$ as the carrier gas. The fatty acid methyl esters (FAMEs) were identified by comparing the retention times of the peaks with those of commercial standards and comparing the respective ion chromatograms with those reported in the NIST 2011 library. The FAMEs were quantified against methyl nonadecanoate, which was added as an internal standard and then quantified using the calibration curves of the respective FAMEs $\left(R^{2} \geq 0.995\right)$. The contents of the FAMEs were expressed as $\mathrm{mg} / 100 \mathrm{~g} \mathrm{FW}$. The experiments were performed in three biological replicates for each accession.

\subsection{Biochemical Properties of Avocado Seed Oil}

The acid, iodine, peroxide, and saponification values were determined from the mixed avocado seed oil (a mixture of the seed oils of 16 avocado accessions), and the standard AOAC methods (Association of Official 
Analytical Chemists, 2000, Method number Cd 3a-63, Cd 1-25, Cd 8-53 and Cd 3-25, respectively) were used. All determinations were carried out in triplicate.

\subsection{Formulation of the Bar Soap}

The self-made bar soap containing the mixed avocado seed oil was made via saponification with $\mathrm{NaOH}$. To prepare the soap, $14.11 \mathrm{~g} \mathrm{NaOH}$ was dissolved in $47 \mathrm{~mL}$ purified water and kept at $40{ }^{\circ} \mathrm{C}$. The oil mixture $(86.21$ g) was heated to $80{ }^{\circ} \mathrm{C}$ and added dropwise to the $\mathrm{NaOH}$ solution. Subsequently, the mixture was added to a rotary evaporator to allow oil saponification at $50{ }^{\circ} \mathrm{C}$ for $1 \mathrm{~h}$, after which a pasty liquid appeared. The liquid was poured gradually into a mold and kept at $25^{\circ} \mathrm{C}$ for two weeks.

\subsection{Physicochemical Properties of the Bar Soap}

The $\mathrm{pH}$ values of the self-made bar soap solutions (10\%) in deionized water were measured (S20K, Mettler Toledo Ltd., Shanghai, China) in triplicate after the bar soaps were stored for 15 days under normal conditions and under ultraviolet (UV) irradiation (10 W, $60 \mathrm{lux}, 365 \mathrm{~nm}$ ). A commercial soap (Nice, Shanghai, China) stored under normal conditions was tested in the same way. The avocado seed oil bar soap (1.0 g) was added to a 100 -mL glass measuring cylinder containing $50 \mathrm{~mL}$ deionized water and shaken vigorously for $2 \mathrm{~min}$ to generate foam. The height of the foam generated was determined immediately following shaking and after 10, 20,30, 40, 50 , and $60 \mathrm{~min}$. The foamability tests were repeated in triplicate under normal conditions and under UV irradiation (10 W, 60 lux, $365 \mathrm{~nm}$ ). A commercial soap (Nice, Shanghai, China) was tested in the same way under normal conditions.

\subsection{Statistical Analysis}

All determinations were carried out in three measurements and the results are given as means \pm standard deviation. The data were analyzed using SPSS version 20.0 (SPSS Inc., Chicago, IL, USA). Significant differences among the fruit characteristics, oil contents, and fatty acid compositions of the 16 avocado accessions were verified by one-way analysis of variance, and Duncan's multiple comparison test was used to determine the statistical significance of differences between means at a $95 \%$ confidence level.

\section{Results and Discussion}

\subsection{Total Lipid Contents and Fatty Acid Compositions of Avocado Seed Identification}

The total lipid contents of the seeds of the 16 avocado accessions are presented in Table 1, indicating significant differences between most of the accessions $(p<0.05)$. Hass had the highest total seed lipid content $(4.75 \mathrm{~g} / 100 \mathrm{~g}$ FW), followed by DL-2 (2.05 g/100 g FW). The seed lipid contents of the remaining 14 avocado accessions were lower, ranging from 0.99 to $1.78 \mathrm{~g} / 100 \mathrm{~g} \mathrm{FW}$. The seed lipid contents determined in the present study were in accordance with the results of Galvão et al. (2014) and Rodríguez-Carpena et al. (2011), but the Hass seed lipid content measured herein was much lower than that determined by Vinha et al. (2013) (14.7 g/100 g FW). The variation of the total lipid levels of the seeds of the avocado cultivars from around the world could be due to the different cultivation conditions, varieties, or mature degrees of fruits. 
Table 1. Total lipid contents (mean value \pm standard deviation, g/100 g FW, $n=3$ ) of the seeds of 16 avocado accessions grown in southern China

\begin{tabular}{ll}
\hline Accession & Total lipid content \\
\hline RN-1 & $1.60 \pm 0.04 \mathrm{a}$ \\
RN-5 & $0.99 \pm 0.01 \mathrm{~b}$ \\
RN-11 & $1.76 \pm 0.00 \mathrm{~d}$ \\
RN-12 & $1.78 \pm 0.01 \mathrm{~d}$ \\
RN-15 & $1.28 \pm 0.00 \mathrm{e}$ \\
RN-16 & $1.37 \pm 0.01 \mathrm{f}$ \\
Hass & $4.75 \pm 0.05 \mathrm{~g}$ \\
DL-1 & $1.51 \pm 0.02 \mathrm{~h}$ \\
DL-2 & $2.05 \pm 0.02 \mathrm{i}$ \\
DL-3 & $1.69 \pm 0.03 \mathrm{j}$ \\
DL-4 & $1.04 \pm 0.02 \mathrm{c}$ \\
RLW-1 & $1.04 \pm 0.01 \mathrm{c}$ \\
RLW-2 & $1.18 \pm 0.06 \mathrm{k}$ \\
RLW-3 & $1.29 \pm 0.06 \mathrm{e}$ \\
RLW-4 & $1.02 \pm 0.00 \mathrm{bc}$ \\
GY-8 & $1.07 \pm 0.01 \mathrm{c}$ \\
\hline
\end{tabular}

Note. Different letters within the column are significantly different $(p<0.05)$.

The fatty acid compositions of the seeds of the 16 avocado accessions are provided in Table 2, 3. The same 17 fatty acids were detected in the seeds of all 16 avocado accessions, although significant differences in composition were observed among most of the accessions $(p<0.05)$. The major fatty acids $(\geq 15 \%$, the percentage of the individual fatty acid out of the total fatty acid content) quantified in avocado seed oil in the present study were linoleic acid (40.14\%), palmitic acid (23.54\%), and oleic acid (16.23\%). Intermediate amounts $(1 \%$ to $15 \%$, the percentage of the individual fatty acid out of the total fatty acid content) of palmitoleic acid $(5.78 \%)$, linolenic acid (4.63\%), stearic acid (3.01\%), and myristic acid $(1.76 \%)$ were detected, and small amounts $(\leq 1 \%$, the percentage of the individual fatty acid out of the total fatty acid content) of the remaining 10 fatty acids were found. These results were in accordance with the previous study showing that linoleic, palmitic, and oleic acids were the predominant fatty acids in avocado seed oil (Galvão et al., 2014). The content of linoleic acid was slightly higher than those of palmitic acid and oleic acid in this study, which was basically in agreement with the results of Galvão et al. (2014). In the present study, $69 \%$ of TFA were unsaturated, while the remaining $31 \%$ were saturated (Table 4). TFA varied from $949.86 \pm 26.40 \mathrm{mg} / 100 \mathrm{~g} \mathrm{FW}$ in RLW-4 to $4677.33 \pm 108.45$ $\mathrm{mg} / 100 \mathrm{~g} \mathrm{FW}$ in Hass. $\Sigma$ UFA ranged from $615.27 \pm 11.22 \mathrm{mg} / 100 \mathrm{~g} \mathrm{FW}$ in RN-5 to $3263.69 \pm 26.48 \mathrm{mg} / 100 \mathrm{~g} \mathrm{FW}$ in Hass, while $\Sigma$ SFA varied between $270.59 \pm 10.27 \mathrm{mg} / 100 \mathrm{~g}$ FW in RLW-4 to $1413.64 \pm 81.97 \mathrm{mg} / 100 \mathrm{~g}$ FW in Hass. The palmitic acid was most abundant fatty acid, with noticeable differences in palmitic acid content between samples $(p<0.05)$. The greatest palmitic acid content was observed in Hass $(1253.96 \mathrm{mg} / 100 \mathrm{~g} \mathrm{FW})$, while the lowest content was found in RLW-4 (192.67 mg/100 g FW). Among UFAs, linoleic acid was the most abundant, with contents ranging from 354.80 (RN-5) to 1497.57 (Hass) mg/100 g FW. The $\Sigma$ UFA/ $\Sigma$ SFA ratios varied from 1.81 to 2.62, which were in accordance with the results of Galvão et al. (2014) (Table 4). 
Table 2. The major and intermediate fatty acids compositions (mean value \pm standard deviation, $\mathrm{mg} / 100 \mathrm{~g} \mathrm{FW}, n$ $=3$ ) in the seeds of 16 avocado accessions grown in southern China

\begin{tabular}{|c|c|c|c|c|c|c|c|c|c|c|c|c|c|c|c|c|}
\hline \multirow{2}{*}{ Fatty Acid } & \multicolumn{16}{|c|}{ Accession } \\
\hline & $\mathrm{RN}-1$ & RN-5 & RN-11 & $\mathrm{RN}-12$ & $\mathrm{RN}-15$ & RN-16 & Hass & DL-1 & DL-2 & DL-3 & DL-4 & RLW-1 & RLW-2 & RLW-3 & RLW-4 & GY-8 \\
\hline \multicolumn{17}{|c|}{ Saturated Fatty Acids (SFAs) } \\
\hline \multirow{2}{*}{$\begin{array}{l}\text { Myristic } \\
\text { acid (C14:0) }\end{array}$} & $27.95 \pm$ & $22.00 \pm$ & $26.03 \pm$ & $37.39 \pm$ & $28.03 \pm$ & $25.93 \pm$ & $37.10 \pm$ & $28.52 \pm$ & $32.37 \pm$ & $45.76 \pm$ & $25.83 \pm$ & $20.77 \pm$ & $21.79 \pm$ & $20.06 \pm$ & $18.80 \pm$ & $22.85 \pm$ \\
\hline & $0.32 \mathrm{a}$ & $0.44 \mathrm{be}$ & $0.93 \mathrm{f}$ & $0.95 \mathrm{~g}$ & $1.01 \mathrm{a}$ & $1.16 \mathrm{f}$ & $1.61 \mathrm{~g}$ & $1.14 \mathrm{a}$ & $0.39 \mathrm{~h}$ & $2.40 \mathrm{i}$ & $1.14 \mathrm{f}$ & $0.67 \mathrm{bc}$ & $0.54 \mathrm{bce}$ & $0.36 \mathrm{~cd}$ & $0.52 \mathrm{~d}$ & $0.48 \mathrm{e}$ \\
\hline \multirow{2}{*}{$\begin{array}{l}\text { Palmitic } \\
\text { acid (C16:0) }\end{array}$} & 337.69 & 250.99 & 492.56 & 393.72 & 288.07 & 359.00 & 1253.96 & 328.46 & 449.95 & 353.04 & 244.23 & 219.77 & 220.61 & 290.48 & 192.67 & 221.02 \\
\hline & $\pm 1.38 \mathrm{a}$ & $\pm 6.13 \mathrm{c}$ & $\pm 20.51 \mathrm{e}$ & $\pm 18.81 \mathrm{~b}$ & $\pm 9.28 \mathrm{f}$ & $\pm 3.12 \mathrm{ab}$ & $\pm 78.12 \mathrm{~g}$ & $\pm 13.48 \mathrm{a}$ & $\pm 4.23 \mathrm{~h}$ & $\pm 6.42 \mathrm{a}$ & $\pm 16.07 \mathrm{c}$ & $\pm 5.20 \mathrm{~cd}$ & $\pm 7.45 \mathrm{~cd}$ & $\pm 3.72 \mathrm{f}$ & $\pm 8.17 \mathrm{~d}$ & $\pm 7.51 \mathrm{~cd}$ \\
\hline \multirow{2}{*}{$\begin{array}{l}\text { Stearic } \\
\text { acid (C18:0) }\end{array}$} & $46.32 \pm$ & $42.40 \pm$ & $46.32 \pm$ & $66.30 \pm$ & $44.92 \pm$ & $46.31 \pm$ & $79.42 \pm$ & $47.15 \pm$ & $51.28 \pm$ & $54.04 \pm$ & $39.90 \pm$ & $39.10 \pm$ & $40.76 \pm$ & $41.13 \pm$ & $35.65 \pm$ & $33.47 \pm$ \\
\hline & $0.71 \mathrm{ab}$ & $0.45 \mathrm{c}$ & $0.53 \mathrm{ab}$ & $1.44 \mathrm{f}$ & $0.28 \mathrm{a}$ & $0.65 \mathrm{ab}$ & $1.31 \mathrm{~g}$ & $0.83 b$ & $0.88 \mathrm{~h}$ & $1.25 \mathrm{i}$ & $0.84 \mathrm{de}$ & $1.76 \mathrm{e}$ & $0.24 \mathrm{cde}$ & $0.92 \mathrm{~cd}$ & $1.10 \mathrm{j}$ & $0.74 \mathrm{k}$ \\
\hline \multicolumn{17}{|c|}{ Unsaturated Fatty Acids (UFAs) } \\
\hline \multirow{2}{*}{$\begin{array}{l}\text { Palmitoleic } \\
\text { acid (16:1) }\end{array}$} & $63.27 \pm$ & $60.44 \pm$ & $89.40 \pm$ & $70.37 \pm$ & $54.00 \pm$ & $48.32 \pm$ & $580.62 \pm$ & $54.09 \pm$ & $76.72 \pm$ & $56.53 \pm$ & $38.39 \pm$ & $52.86 \pm$ & $55.93 \pm$ & $73.84 \pm$ & $40.58 \pm$ & $33.40 \pm$ \\
\hline & $1.76 \mathrm{a}$ & $3.07 \mathrm{ab}$ & $1.96 \mathrm{~d}$ & $3.06 \mathrm{e}$ & $1.64 \mathrm{bc}$ & $0.34 \mathrm{c}$ & $15.20 \mathrm{f}$ & $1.62 \mathrm{bc}$ & $0.97 \mathrm{e}$ & $0.88 \mathrm{ab}$ & $0.90 \mathrm{~g}$ & $1.02 \mathrm{bc}$ & $2.24 \mathrm{abc}$ & $1.50 \mathrm{e}$ & $0.35 \mathrm{~g}$ & $0.87 \mathrm{~g}$ \\
\hline \multirow{2}{*}{$\begin{array}{l}\text { Oleic acid } \\
(\mathrm{C} 18: 1)\end{array}$} & 179.85 & 160.58 & 342.58 & 253.38 & 266.08 & 174.51 & 1070.98 & 202.49 & 276.01 & 177.53 & 139.93 & 126.29 & 209.35 & 220.10 & 156.33 & 109.57 \\
\hline & $\pm 3.38 \mathrm{a}$ & $\pm 1.27 \mathrm{~b}$ & $\pm 5.66 \mathrm{c}$ & $\pm 7.53 \mathrm{~d}$ & $\pm 1.34 \mathrm{e}$ & $\pm 0.17 \mathrm{a}$ & $\pm 7.87 \mathrm{f}$ & $\pm 5.56 \mathrm{~g}$ & $\pm 5.11 \mathrm{~h}$ & $\pm 1.70 \mathrm{a}$ & $\pm 1.61 \mathrm{i}$ & $\pm 1.15 \mathrm{j}$ & $\pm 6.59 \mathrm{~g}$ & $\pm 6.33 \mathrm{k}$ & $\pm 6.50 \mathrm{~b}$ & \pm 3.171 \\
\hline \multirow{2}{*}{$\begin{array}{l}\text { Linoleic } \\
\text { acid (C18:2) }\end{array}$} & 594.92 & 354.80 & 579.50 & 687.49 & 420.24 & 626.84 & 1497.57 & 711.32 & 966.26 & 845.22 & 465.78 & 466.84 & 454.37 & 536.61 & 413.25 & 433.65 \\
\hline & $\pm 8.76 \mathrm{a}$ & $\pm 5.73 \mathrm{~b}$ & $\pm 4.80 \mathrm{c}$ & $\pm 4.73 \mathrm{~d}$ & $\pm 6.79 \mathrm{e}$ & $\pm 7.44 \mathrm{f}$ & $\pm 2.14 \mathrm{~g}$ & $\pm 3.97 \mathrm{~h}$ & $\pm 6.23 \mathrm{i}$ & $\pm 7.30 \mathrm{j}$ & $\pm 8.32 \mathrm{k}$ & $\pm 4.46 \mathrm{k}$ & \pm 9.161 & $\pm 5.17 \mathrm{~m}$ & $\pm 8.16 \mathrm{e}$ & $\pm 7.69 \mathrm{n}$ \\
\hline \multirow{2}{*}{$\begin{array}{l}\text { Linolenic } \\
\text { acid (C18:3) }\end{array}$} & 109.01 & $38.83 \pm$ & $61.20 \pm$ & $71.52 \pm$ & $74.37 \pm$ & $61.30 \pm$ & 113.15 & $64.29 \pm$ & 101.35 & 109.62 & $47.53 \pm$ & $51.96 \pm$ & $60.15 \pm$ & $64.19 \pm$ & $68.70 \pm$ & $63.58 \pm$ \\
\hline & $\pm 0.58 \mathrm{a}$ & $1.14 \mathrm{~b}$ & $1.33 \mathrm{~cd}$ & $2.97 \mathrm{ef}$ & $1.85 \mathrm{e}$ & $2.68 \mathrm{~cd}$ & $\pm 1.24 \mathrm{~g}$ & $2.06 \mathrm{~d}$ & $\pm 4.43 \mathrm{~h}$ & $\pm 0.14 \mathrm{a}$ & $1.90 \mathrm{i}$ & $1.81 \mathrm{j}$ & $0.83 \mathrm{c}$ & $1.76 \mathrm{~d}$ & $1.08 \mathrm{f}$ & $1.24 \mathrm{~cd}$ \\
\hline
\end{tabular}

Note. Different letters within the same row are significantly different $(p<0.05) ; \Sigma \mathrm{SFA}=$ total saturated fatty acids; $\Sigma U F A=$ total unsaturated fatty acids; TFA $=$ total fatty acids.

Table 3. The small amounts of fatty acids compositions (mean value \pm standard deviation, $\mathrm{mg} / 100 \mathrm{~g} \mathrm{FW}, n=3$ ) in the seeds of 16 avocado accessions grown in southern China

\begin{tabular}{|c|c|c|c|c|c|c|c|c|c|c|c|c|c|c|c|c|}
\hline \multirow{2}{*}{ Fatty Acid } & \multicolumn{16}{|c|}{ Accession } \\
\hline & $\mathrm{N}-1$ & $\mathrm{RN}-5$ & RN-11 & RN-12 & $\mathrm{RN}$ & $\mathrm{RN}$ & Hass & DL-1 & DL-2 & DL-3 & L-4 & RL & -2 & RLI & RLI & GY-8 \\
\hline \multicolumn{17}{|c|}{ Saturated Fatty Acids (SFAs) } \\
\hline \multirow{2}{*}{$\begin{array}{l}\text { Capric acid } \\
(\mathrm{C} 10: 0)\end{array}$} & $0.84 \pm$ & $0.21 \pm$ & $0.34 \pm$ & $1.11 \pm$ & $0.46 \pm$ & $0.98 \pm$ & $0.78 \pm$ & $0.75 \pm$ & $0.59 \pm$ & $0.93 \pm$ & $0.73 \pm$ & $0.18 \pm$ & $0.18 \pm$ & $0.27 \pm$ & $0.25 \pm$ & $0.61 \pm$ \\
\hline & $0.03 \mathrm{a}$ & $0.01 \mathrm{bc}$ & $0.02 \mathrm{e}$ & $0.06 \mathrm{f}$ & $0.00 \mathrm{~g}$ & $0.04 \mathrm{~h}$ & $0.04 \mathrm{i}$ & $0.02 \mathrm{i}$ & $0.02 \mathrm{j}$ & $0.05 \mathrm{k}$ & $0.03 \mathrm{i}$ & $0.01 \mathrm{~b}$ & $0.01 \mathrm{~b}$ & $0.01 \mathrm{~d}$ & $0.01 \mathrm{~cd}$ & $0.02 \mathrm{j}$ \\
\hline \multirow{2}{*}{$\begin{array}{l}\text { Hendecanoic } \\
\text { acid (C11:0) }\end{array}$} & $0.61 \pm$ & $0.11 \pm$ & $0.24 \pm$ & $0.52 \pm$ & $0.31 \pm$ & $0.21 \pm$ & $0.94 \pm$ & $0.65 \pm$ & $0.29 \pm$ & $0.90 \pm$ & $0.54 \pm$ & $0.14 \pm$ & $0.14 \pm$ & $0.14 \pm$ & $0.22 \pm$ & $0.77 \pm$ \\
\hline & $0.02 \mathrm{a}$ & $0.00 \mathrm{~b}$ & $0.01 \mathrm{~d}$ & $0.02 \mathrm{f}$ & $0.01 \mathrm{~g}$ & $0.01 \mathrm{e}$ & $0.02 \mathrm{~h}$ & $0.01 \mathrm{i}$ & $0.01 \mathrm{j}$ & $0.02 \mathrm{k}$ & $0.01 \mathrm{f}$ & $0.01 \mathrm{bc}$ & $0.01 \mathrm{c}$ & $0.01 \mathrm{bc}$ & $0.00 \mathrm{de}$ & 0.021 \\
\hline \multirow{2}{*}{$\begin{array}{l}\text { Lauric acid } \\
(\mathrm{C} 12: 0)\end{array}$} & $1.41 \pm$ & $0.56 \pm$ & $1.09 \pm$ & $4.61 \pm$ & $6.76 \pm$ & $1.96 \pm$ & $3.48 \pm$ & $2.93 \pm$ & $2.32 \pm$ & $4.04 \pm$ & $3.36 \pm$ & $1.02 \pm$ & $0.96 \pm$ & $1.44 \pm$ & $1.77 \pm$ & $1.93 \pm$ \\
\hline & $0.03 a$ & $0.01 \mathrm{~b}$ & $0.04 \mathrm{c}$ & $0.11 \mathrm{e}$ & $0.06 \mathrm{f}$ & $0.04 \mathrm{~g}$ & $0.08 \mathrm{~h}$ & $0.09 \mathrm{i}$ & $0.05 \mathrm{j}$ & $0.07 \mathrm{k}$ & 0.081 & $0.06 \mathrm{~cd}$ & $0.06 \mathrm{~d}$ & $0.02 \mathrm{a}$ & $0.07 \mathrm{~m}$ & $0.06 \mathrm{~g}$ \\
\hline \multirow{2}{*}{$\begin{array}{l}\text { Tridecylic } \\
\text { acid (C13:0) }\end{array}$} & $2.66 \pm$ & $1.16 \pm$ & $2.24 \pm$ & $5.33 \pm$ & $3.04 \pm$ & $1.78 \pm$ & $7.15 \pm$ & $3.31 \pm$ & $2.36 \pm$ & $5.21 \pm$ & $4.15 \pm$ & $3.44 \pm$ & $3.23 \pm$ & $3.70 \pm$ & $6.57 \pm$ & $2.94 \pm$ \\
\hline & $0.06 \mathrm{a}$ & $0.01 \mathrm{~b}$ & $0.09 \mathrm{c}$ & $0.07 \mathrm{~d}$ & $0.07 \mathrm{e}$ & $0.06 f$ & $0.06 \mathrm{~g}$ & $0.08 \mathrm{hi}$ & $0.08 \mathrm{c}$ & $0.03 \mathrm{~d}$ & $0.04 \mathrm{j}$ & $0.21 \mathrm{~h}$ & $0.01 \mathrm{i}$ & $0.10 \mathrm{k}$ & 0.081 & $0.02 \mathrm{e}$ \\
\hline \multirow{2}{*}{$\begin{array}{l}\text { Pentadecanoic } \\
\text { acid (C15:0) }\end{array}$} & $2.20 \pm$ & $0.45 \pm$ & $1.18 \pm$ & $3.35 \pm$ & $1.17 \pm$ & $1.32 \pm$ & $2.54 \pm$ & $1.71 \pm$ & $1.38 \pm$ & $1.57 \pm$ & $1.47 \pm$ & $0.94 \pm$ & $0.88 \pm$ & $1.22 \pm$ & $1.66 \pm$ & $1.25 \pm$ \\
\hline & $0.06 \mathrm{a}$ & $0.01 \mathrm{~b}$ & $0.02 \mathrm{c}$ & $0.04 \mathrm{j}$ & $0.05 \mathrm{c}$ & $0.04 \mathrm{de}$ & $0.10 \mathrm{k}$ & $0.09 \mathrm{i}$ & $0.02 \mathrm{ef}$ & $0.08 \mathrm{gh}$ & $0.04 \mathrm{fg}$ & 0.041 & 0.041 & $0.05 \mathrm{~cd}$ & $0.09 \mathrm{hi}$ & $0.08 \mathrm{~cd}$ \\
\hline \multirow{2}{*}{$\begin{array}{l}\text { Heptadecanoic } \\
\text { acid }(\mathrm{C} 17: 0)\end{array}$} & $48.06 \pm$ & $18.64 \pm$ & $1.81 \pm$ & $64.87 \pm$ & $30.17 \pm$ & $2.00 \pm$ & $1.97 \pm$ & $2.62 \pm$ & $2.67 \pm$ & $2.42 \pm$ & $1.55 \pm$ & $0.72 \pm$ & $0.63 \pm$ & $0.98 \pm$ & $0.81 \pm$ & $1.99 \pm$ \\
\hline & $1.51 \mathrm{a}$ & $0.54 b$ & $0.08 \mathrm{~cd}$ & $1.00 \mathrm{~g}$ & $0.09 \mathrm{~h}$ & $0.04 \mathrm{~cd}$ & $0.03 \mathrm{~cd}$ & $0.00 \mathrm{c}$ & $0.07 \mathrm{c}$ & $0.06 \mathrm{~cd}$ & $0.04 \mathrm{de}$ & $0.07 \mathrm{ef}$ & $0.04 \mathrm{ef}$ & $0.01 \mathrm{ef}$ & $0.03 \mathrm{ef}$ & $0.09 \mathrm{~cd}$ \\
\hline \multirow{2}{*}{$\begin{array}{l}\text { Arachic acid } \\
\text { (C20:0) }\end{array}$} & $1.35 \pm$ & $0.59 \pm$ & $1.09 \pm$ & $2.35 \pm$ & $1.48 \pm$ & $1.27 \pm$ & $6.01 \pm$ & $2.42 \pm$ & $2.48 \pm$ & $2.03 \pm$ & $1.22 \pm$ & $1.16 \pm$ & $0.84 \pm$ & $1.06 \pm$ & $1.24 \pm$ & $1.65 \pm$ \\
\hline & $0.06 \mathrm{a}$ & $0.03 \mathrm{e}$ & $0.06 \mathrm{~d}$ & $0.10 \mathrm{f}$ & $0.04 \mathrm{~h}$ & $0.05 \mathrm{ab}$ & $0.05 \mathrm{i}$ & $0.04 \mathrm{fg}$ & $0.08 \mathrm{~g}$ & $0.09 \mathrm{j}$ & $0.04 b c$ & $0.02 \mathrm{~cd}$ & $0.05 \mathrm{k}$ & $0.03 \mathrm{~d}$ & $0.04 \mathrm{bc}$ & 0.091 \\
\hline \multirow{2}{*}{$\begin{array}{l}\text { Behenic acid } \\
\text { (C22:0) }\end{array}$} & $4.99 \pm$ & $1.48 \pm$ & $2.04 \pm$ & $7.85 \pm$ & $3.42 \pm$ & $6.38 \pm$ & $9.54 \pm$ & $11.80 \pm$ & $8.42 \pm$ & $8.17 \pm$ & $3.11 \pm$ & $2.85 \pm$ & $2.44 \pm$ & $3.34 \pm$ & $3.85 \pm$ & $7.62 \pm$ \\
\hline & $0.04 \mathrm{a}$ & $0.00 \mathrm{~b}$ & $0.05 \mathrm{c}$ & $0.09 \mathrm{~d}$ & $0.12 \mathrm{e}$ & $0.23 \mathrm{~h}$ & $0.31 \mathrm{i}$ & $0.27 \mathrm{j}$ & $0.37 \mathrm{k}$ & $0.04 \mathrm{k}$ & $0.08 \mathrm{fg}$ & $0.09 \mathrm{~g}$ & 0.051 & $0.08 \mathrm{ef}$ & $0.08 \mathrm{~m}$ & $0.17 \mathrm{~d}$ \\
\hline \multirow{2}{*}{$\begin{array}{l}\text { Tricosane } \\
\text { acid }(\mathrm{C} 23: 0)\end{array}$} & $14.70 \pm$ & $3.88 \pm$ & $6.41 \pm$ & $10.60 \pm$ & $7.44 \pm$ & $10.71 \pm$ & $10.75 \pm$ & $13.83 \pm$ & $11.39 \pm$ & $11.04 \pm$ & $6.37 \pm$ & $5.21 \pm$ & $5.24 \pm$ & $7.35 \pm$ & $7.10 \pm$ & $8.87 \pm$ \\
\hline & $0.15 \mathrm{a}$ & $0.15 b$ & $0.29 \mathrm{c}$ & $0.52 \mathrm{~d}$ & $0.08 \mathrm{~g}$ & $0.29 \mathrm{de}$ & $0.24 \mathrm{de}$ & $0.10 \mathrm{~h}$ & $0.17 f$ & $0.47 \mathrm{ef}$ & $0.13 \mathrm{c}$ & $0.15 \mathrm{i}$ & $0.21 \mathrm{i}$ & $0.14 \mathrm{~g}$ & $0.08 \mathrm{~g}$ & $0.07 \mathrm{j}$ \\
\hline \multicolumn{17}{|c|}{ Unsaturated Fatty Acids (UFAs) } \\
\hline \multirow{2}{*}{$\begin{array}{l}\text { Myristoleic } \\
\text { acid (C14:1) }\end{array}$} & $1.88 \pm$ & $0.62 \pm$ & $2.56 \pm$ & $2.46 \pm$ & $2.55 \pm$ & $1.15 \pm$ & $1.37 \pm$ & $3.37 \pm$ & $4.38 \pm$ & $6.41 \pm$ & $2.58 \pm$ & $0.64 \pm$ & $0.61 \pm$ & $0.47 \pm$ & $0.41 \pm$ & $2.06 \pm$ \\
\hline & $0.07 \mathrm{a}$ & $0.01 \mathrm{~b}$ & $0.01 \mathrm{c}$ & $0.06 \mathrm{c}$ & $0.02 \mathrm{c}$ & $0.04 \mathrm{~d}$ & $0.03 \mathrm{e}$ & $0.21 \mathrm{f}$ & $0.14 \mathrm{~g}$ & $0.10 \mathrm{~h}$ & $0.07 \mathrm{c}$ & $0.06 \mathrm{~b}$ & $0.05 \mathrm{~b}$ & $0.03 \mathrm{i}$ & $0.04 \mathrm{i}$ & $0.03 \mathrm{j}$ \\
\hline
\end{tabular}

Note. Different letters within the same row are significantly different $(p<0.05) ; \Sigma \mathrm{SFA}=$ total saturated fatty acids $\Sigma$ UFA $=$ total unsaturated fatty acids; TFA $=$ total fatty acids. 
Table 4. Saturated fatty acids (SFA), unsaturated fatty acids (UFA), and total fatty acids (TFA) profile (mean value \pm standard deviation, $\mathrm{mg} / 100 \mathrm{~g} \mathrm{FW}, n=3$ ) in the seeds of 16 avocado accessions grown in southern China

\begin{tabular}{|c|c|c|c|c|c|c|c|c|c|c|c|c|c|c|c|c|}
\hline \multirow{2}{*}{ Fatty Acid } & \multicolumn{16}{|c|}{ Accession } \\
\hline & RN-1 & RN-5 & $\mathrm{RN}-11$ & RN-12 & RN-15 & RN-16 & Hass & DL-1 & DL-2 & DL-3 & DL-4 & RLW-1 & RLW-2 & RLW-3 & RLW-4 & GY-8 \\
\hline \multirow[t]{2}{*}{$\Sigma$ SFA } & 488.77 & 342.47 & 581.35 & 598.00 & 415.27 & 457.85 & 1413.64 & 444.15 & 565.50 & 489.15 & 332.46 & 295.30 & 297.70 & 371.17 & 270.59 & 310.90 \\
\hline & \pm 4.37 & \pm 7.78 & \pm 22.63 & \pm 23.21 & \pm 11.09 & \pm 5.73 & \pm 81.97 & \pm 16.20 & \pm 6.37 & \pm 10.98 & \pm 18.54 & \pm 8.29 & \pm 8.71 & \pm 5.45 & \pm 10.27 & \pm 9.41 \\
\hline \multirow[t]{2}{*}{$\Sigma \mathrm{UFA}$} & 948.93 & 615.27 & 1075.24 & 1085.22 & 817.24 & 912.12 & 3263.69 & 1035.56 & 1424.72 & 1195.31 & 694.21 & 698.59 & 780.41 & 895.21 & 679.27 & 642.26 \\
\hline & \pm 14.55 & \pm 11.22 & \pm 13.76 & \pm 18.35 & \pm 11.64 & \pm 10.67 & \pm 26.48 & \pm 13.42 & \pm 16.88 & \pm 10.12 & \pm 12.80 & \pm 8.50 & \pm 18.87 & \pm 14.79 & \pm 16.13 & \pm 13.00 \\
\hline \multirow[t]{2}{*}{ TFA } & 1437.70 & 957.74 & 1656.58 & 1683.22 & 1232.51 & 1369.97 & 4677.33 & 1479.71 & 1990.22 & 1684.46 & 1026.67 & 993.89 & 1078.11 & 1266.38 & 949.86 & 953.16 \\
\hline & \pm 18.00 & \pm 19.00 & \pm 36.39 & \pm 41.56 & \pm 22.73 & \pm 16.40 & \pm 108.45 & \pm 29.62 & \pm 23.25 & \pm 21.10 & \pm 31.34 & \pm 16.79 & \pm 27.58 & \pm 20.24 & \pm 26.40 & \pm 2.41 \\
\hline$\Sigma U F A / \Sigma S F A$ & 1.94 & 1.80 & 1.85 & 1.81 & 1.97 & 1.99 & 2.31 & 2.33 & 2.52 & 2.44 & 2.09 & 2.37 & 2.62 & 2.41 & 2.51 & 2.07 \\
\hline
\end{tabular}

Note. Different letters within the same row are significantly different $(p<0.05) ; \Sigma \mathrm{SFA}=$ total saturated fatty acids; $\Sigma U F A=$ total unsaturated fatty acids; TFA $=$ total fatty acids.

\subsection{Confirmation of Biochemical Properties of Avocado Seed Oil}

The biochemical properties of avocado seed oil were evaluated and compared with those of unconventional seed bio-oils (Table 5). These properties were vital for the application of crude extracted oil in cosmetics (Lourith et al., 2014). A lower acid value corresponds to a better quality of bio-oil (Wu et al., 2015). The acid value of avocado seed oil was higher than that of avocado and mango seed oil determined by Galvão et al. (2014) and Wu et al. (2015), but lower than those of other two seed bio-oils (Lourith et al., 2016; Warra, 2016). The iodine value of avocado seed oil detected in the present study exceeds those of other seed bio-oils except for that of pumpkin seed oil (Table 5). This high iodine value implies an enhanced degree of oxidation or hydrogenation resulting from the degree of UFAs (Wu et al., 2015), when the oil was stored in the sealed state. That displayed that the seeds of avocado contained higher unsaturated fatty acid compositions than other seed bio-oils. Similarly, the peroxide value also has the positive correlation with unsaturated fatty acid content (Lourith et al., 2014; Warra, 2016). The avocado seed oil in the present study had the same peroxide value as Para rubber and Neocarya macrophylla seed oils (Lourith et al., 2014; Warra, 2016). The saponification value of the avocado seed oil was lower than most of the other seed bio-oils (Table 5). The saponification value in the present study indicated that the proportion of long-chain or great-molecular-weight fatty acids in the avocado seed oil had more than twice than those of the majority of seed bio-oils (Table 5). This saponification value was in accordance with the fatty acid profiles shown in Table 2, 3, which presented that the major fatty acids quantified in avocado seed oil were long-chain fatty acids (linoleic acid, palmitic acid, and oleic acid).

Table 5. Physicochemical properties of avocado seed oil and other crops seed oils (mean value \pm standard deviation, $n=3$ )

\begin{tabular}{|c|c|c|c|c|c|}
\hline Species & $\begin{array}{l}\mathrm{AV} \\
\text { (mg KOH/g oil) }\end{array}$ & $\begin{array}{l}\text { IV } \\
\text { (g I } / 100 \text { g oil) }\end{array}$ & $\begin{array}{l}\mathrm{PV} \\
\left(\mathrm{meq} \mathrm{H}_{2} \mathrm{O}_{2}\right)\end{array}$ & $\begin{array}{l}\mathrm{SV} \\
\text { (mg KOH/g oil) }\end{array}$ & Reference \\
\hline Avocado seed oil & $3.74 \pm 0.06$ & $124.09 \pm 1.14$ & $49.83 \pm 0.76$ & $167.98 \pm 10.75$ & Present study \\
\hline Avocado seed oil & $\begin{array}{l}(1.19 \pm 0.01)- \\
(2.23 \pm 0.10)\end{array}$ & $\begin{array}{l}(62.09 \pm 0.35)- \\
(68.53 \pm 1.92)\end{array}$ & - & $\begin{array}{l}(206.7 \pm 1.61)- \\
(261.5 \pm 3.17)\end{array}$ & Galvão et al. (2014) \\
\hline Dalbergia odorifera seed oil & - & $106.53 \pm 0.53$ & - & $196.78 \pm 0.61$ & Zheng et al. (2012) \\
\hline Date plum seed oil & - & $76.66 \pm 0.28$ & - & $191.28 \pm 0.50$ & Nehdi et al. (2010) \\
\hline Mango seed oil & 3.4 & 45.9 & - & 185.6 & Wu et al. (2015) \\
\hline Moringa oleifera seed oil & - & $69.45 \pm 1.20$ & - & $186.67 \pm 2.01$ & Anwar et al. (2003) \\
\hline Neocarya macrophylla seed oil & $12.97 \pm 0.01$ & $32.07 \pm 0.01$ & $45.48 \pm 0.02$ & $153.30 \pm 0.10$ & Warra (2016) \\
\hline Para rubber seed oil & - & $42.34 \pm 0.43$ & $47.33 \pm 0.22$ & $149.25 \pm 1.56$ & Lourith et al. (2014) \\
\hline Pumpkin seed oil & - & $153.66 \pm 0.65$ & - & $175.00 \pm 1.30$ & Rezig et al. (2012) \\
\hline Rambutan seed oil & $3.95 \pm 0.06$ & $47.00 \pm 0.20$ & - & $186.00 \pm 1.00$ & Soís-Fuentes et al. (2010) \\
\hline Rambutan seed oil & - & $50.30 \pm 1.24$ & - & $182.10 \pm 0.16$ & Manaf et al. (2013) \\
\hline Rambutan seed oil & $4.35 \pm 0.00$ & $44.17 \pm 0.30$ & - & $246.73 \pm 0.10$ & Lourith et al. (2016) \\
\hline
\end{tabular}

Note. AV: acid value, IV: iodine value, PV: peroxide value, SV: saponification value. 


\subsection{Determination of Physicochemical Properties of the Bar Soap}

Fats and oil are primary raw materials of cosmetic detergent products owing to alkaline saponification of the fatty acid constituents (Wu et al., 2015; Lourith et al., 2016). The level of alkalinity for cosmetic detergent products is directly related to skin sensitivity (Lourith et al., 2016). The alkalinity of the self-made bar soap stored for 15 days under normal conditions was higher than that of the commercial soap. During UV irradiation, the alkalinity of the self-made bar soap solution decreased and ultimately became lower than that of the commercial soap after 15 days (Figure 1). However, the alkalinities of the self-made bar soap solutions were within the range of soap Chinese national standard values for the commercial bar soap after storage for 15 days under normal conditions or under UV irradiation (QB/T 2485-2000, 2001). Lourith et al. (2016) suggested that the alkalinity of the freshly prepared bar soaps containing rambutan seed oil was within the range from 10.39 to 10.54 , and the alkalinity slightly decreased after the bar soaps were stored under ambient condition for 15 days (Lourith et al., 2016). In this experiment, the alkalinity (9.48) of the self-made bar soap solution was slightly lower than that (10.33 and 10.48) of rambutan seed oil bar soap solution (Lourith et al., 2016), when the avocado seed oil and rambutan seed oil bar soaps were all stored for 15 days under normal conditions.

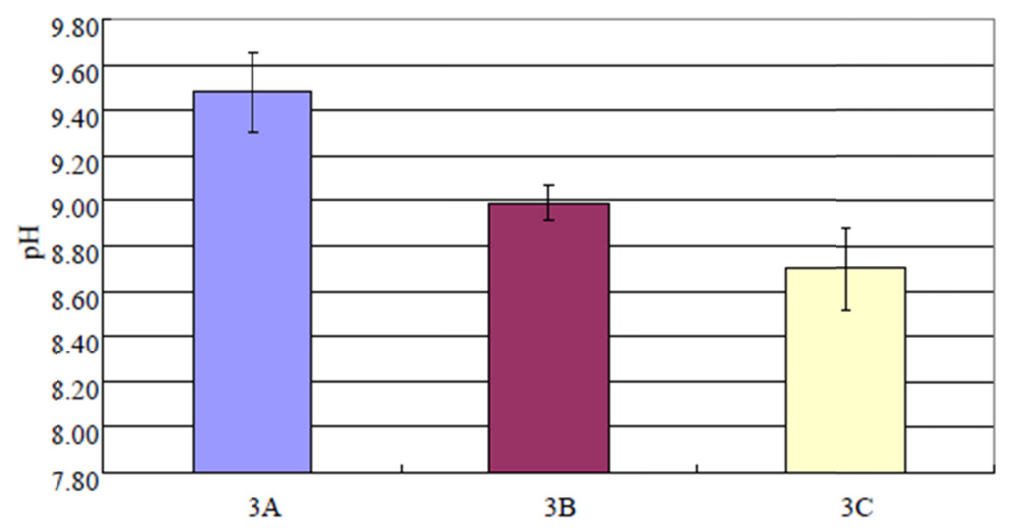

Figure 1. The $\mathrm{pH}$ (mean value \pm standard deviation, $n=3$ ) of the self-made bar soap solution. The self-made bar soap stored for 15 days under normal conditions (1A); the commercial soap solution under normal conditions

(1B); the self-made bar soap solution stored for 15 days under UV irradiation $(10 \mathrm{~W})(1 \mathrm{C})$. Vertical bars represent standard deviations

Foamability is one of the most important properties for cosmetic detergent products (Wu et al., 2015; Lourith et al., 2016). The foaming property of the self-made bar soap solution was tested and compared with that of the commercial soap solution. The foam height of the self-made bar soap solution progressively decreased from the beginning of storage to $60 \mathrm{~min}$ (Figure 2). This phenomenon was also observed by Wu et al. (2015) and Lourith et al. (2016). In addition, the foam height of the self-made bar soap solution was lower than that of the commercial soap solution at all times. Wu et al. (2015) reported that the foam height of mango seed oil bar soap solution was higher than that of a commercial soap solution for the first three hours of storage but then became lower than that of the commercial soap solution (Wu et al., 2015). In this experiment, we could not aware of the specific components in the selected commercial soap, which concerned trade secrets. We speculated that some chemical additives such as liquid paraffin, squalane and squalene were included in the commercial soap, which might enhance the foaming properties (Shrestha et al., 2006; Qin et al., 2013). The foamability of the self-made bar soap solution was not different under normal conditions and under UV irradiation, indicating that UV irradiation did not greatly affect the foamability. 


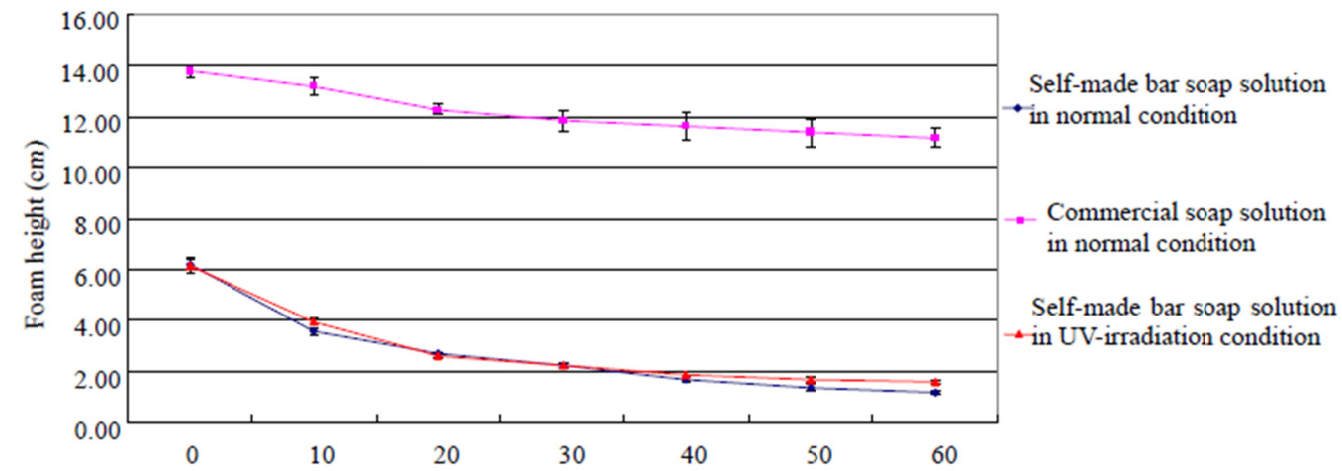

Figure 2. The foamability testing of the self-made bar soap solution (mean value \pm standard deviation, $n=3$ ). Vertical bars represent standard deviations

\section{Conclusions}

Oil contents and fatty acid compositions of the seeds of 16 avocado accessions collected from southern China and their application in cosmetics have been studied. The seeds of 16 avocado accessions differed in oil content and fatty acid composition. Linoleic (40.14\%), palmitic (23.54\%), and oleic acids (16.23\%) were the dominating fatty acids in the avocado seed. Avocado seed oil showed superior biochemical properties than some other seed bio-oils and displayed great promise as a raw material in the cosmetic industry. The bar soap containing avocado seed oil was made. Therefore, transforming of the seed oil that is by-product into cosmetics is encouraged.

\section{Acknowledgements}

The research is financed by the National Natural Science Foundation of China (Grant No. 31701883) and the Central Public-interest Scientific Institution Basal Research Fund for Chinese Academy of Tropical Agricultural Sciences (Grant No. 1630092018003).

\section{References}

Anwar, F., \& Bhanger, M.I. (2003) Analytical characterization of Moringa oleifera seed oil grown in temperate regions of Pakistan. Journal of Agricultural Food Chemistry, 51, 6558-6563. https://doi.org/10.1021/ jf0209894

Dreher, M. L., \& Davenport, A. J. (2013). Hass avocado composition and potential health effects. Critical Reviews in Food Science and Nutrition, 53, 738-750. https://doi.org/10.1080/10408398.2011.556759

FAOSTAT. (2018). Retrieved July 11, 2018, from http://www.fao.org/faostat/en/\#data/QC

Galvão, M. D. S., Narain, N., \& Nigam, N. (2014). Influence of different cultivars on oil quality and chemical characteristics of avocado fruit. Food Science and Technology, Campinas, 34, 539-546. https://doi.org/ $10.1590 / 1678-457 x .6388$

Ge, Y., Ma, F. N., Wu, B., \& Tan, L. (2018). Morphological and chemical analysis of 16 avocado accessions (Persea americana) from China by principal component analysis and cluster analysis. Journal of Agricultural Science, 10, 80-89. https://doi.org/10.5539/jas.v10n8p80

Ge, Y., Si, X. Y., Cao, J. Q., Zhou, Z. X., Wang, W. L., \& Ma, W. H. (2017b). Morphological characteristics, nutritional quality, and bioactive constituents in fruits of two avocado (Persea americana) varieties from hainan province, China. Journal of Agricultural Science, 9, 8-17. https://doi.org/10.5539/jas.v9n2p8

Ge, Y., Si, X. Y., Lin, X. E., Wang, J. S., Zang, X. P., \& Ma, W. H. (2017a). Advances in avocado (Persea americana Mill.). China South Fruit, 46, 63-70.

Giraldoa, L., \& Moreno-Piraján, J. C. (2012). Lipase supported on mesoporous materials as a catalyst in the synthesis of biodiesel from Persea americana mill. oil. Journal of Molecular Catalysis B: Enzymatic, 77, 32-38. https://doi.org/10.1016/j.molcatb.2012.01.001

Knothe, G. (2013). Avocado and olive oil methyl esters. Biomass Bioenergy, 58, 143-148. https://doi.org/ 10.1016/j.biombioe.2013.09.003 
Lourith, N., Kanlayavattanakul, M., Mongkonpaibool, K., Butsaratrakool, T., \& Chinmuang, T. (2016). Rambutan seed as a new promising unconventional source of specialty fat for cosmetics. Indusial Crop Products, 83, 149-154. https://doi.org/10.1016/j.indcrop.2015.12.045

Lourith, N., Kanlayavattanakul, M., Sucontphunt, A., \& Ondee, T. (2014). Para rubber seed oil: New promising unconventional oil for cosmetics. Journal of Oleo Science, 63, 709-716. https://doi.org/10.5650/ jos.ess 14015

Manaf, Y. N. A., Marikkar, J. M. N., Long, K., \& Ghazali, H. M. (2013). Physico-chemical characterization of the fat from red-skin rambutan (Nephellium lappaceum L) seed. Journal of Oleo Science, 62, 335-343. https://doi.org/10.5650/jos.62.335

Medina-Carrillo, R. E., Salazar-Garcia, S., Bonilla-Cardenas, J. A., Herrera-Gonzalez, J. A., Ibarra-Estrada, M. E., \& Alvarez-Bravo, A. (2017). Secondary metabolites and lignin in 'Hass' avocado fruit skin during fruit development in three producing regions. Hortscience, 52, 852-858. https://doi.org/10.21273/HORTSC I11882-17

Nehdi, I., Omri, S., Khalil, M. I., \& Al-Resayes, S. I. (2010). Characteristics and chemical composition of date plum (Phoenix canariensis) seeds and seed oil. Indusial Crop Products, 32, 360-365. https://doi.org/ 10.1016/j.indcrop.2010.05.016

Papademetriou, M. K. (2013). Avocado Production in Asia and the Pacific. Bangkok, BK: FAO Publisher.

QB/T 2485-2000. (2001). Bar soap, laundry bar soap, and compound laundry bar soap.

Qin, S. Q., Hansen, B. B., \& Kiil, S. (2013). Foaming in wet flue gas desulfurization plants: Laboratory-scale investigation of long-term performance of antifoaming agents. AlChE Jounal, 59, 3741-3747. https://doi.org/10.1002/aic.14135

Rezig, L., Chouaibi, M., Msaada, K., \& Hamdi, S. (2012). Chemical composition and profile characterization of pumpkin (Cucurbita maxima) seed oil. Indusial Crop Products, 37, 82-97. https://doi.org/10.1016/j.indcrop. 2011.12.004

Rodríguez-Carpena, J. G., Morcuende, D., Andrade, M. J., Kylli, P., \& Estevez, M. (2011). Avocado (Persea americana Mill.) phenolics, in vitro antioxidant and antimicrobial activities, and inhibition of lipid and protein oxidation in porcine patties. Journal of Agricultural Food Chemistry, 59, 5625-5635. https://doi.org/ $10.1021 / \mathrm{jf1} 1048832$

Santo, M. A. Z., Alicieo, T. V. R., Pereira, C. M. P., Ramis-Ramos, G., \& Mendonca, C. R. B. (2014). Profile of bioactive compounds in avocado pulp oil: Influence of the drying processes and extraction methods. Journal of the American Oil Chemists Society, 91, 19-27. https://doi.org/10.1007/s11746-013-2289-x

Schaffer, B., Wolstenholme, B. N., \& Whiley, A. W. (2012). The Avocado: Botany, Production and Uses (2nd ed.). Croydon, CD: CPI Group (UK) Ltd.

Shrestha, L. K., Aramaki, K., \& Kato, H. (2006). Foaming properties of monoglycerol fatty acid esters in nonpolar oil systems. Langmuir, 22, 8337-8345. https://doi.org/10.1021/la061204h

Solís-Fuentes, J. A., Camey-Ortíz, G., del Rosario, H. M. M., Pérez-Mendoza, F., \& Durán-de-Bazúa, C. (2010). Composition, phase behavior and thermal stability of natural edible fat from rambutan (Nephelium lappaceum L.) seed. Bioresource Technology, 101, 799-803. https://doi.org/10.1016/j.biortech.2009.08.031

Vinha, A. F., Moreira, J., \& Barreira, S. V. P. (2013). Physicochemical parameters, phytochemical composition and antioxidant activity of the Algarvian avocado (Persea americana Mill.). Journal of Agricultural Science, 5, 100-109. https://doi.org/10.5539/jas.v5n12p100

Warra, A. A. (2016). Soap production from quality assessed gingerbread plum (Neocarya Macrophylla) seed oil. Journal of Science Research in Pharmaceutical, Biological and Chemical Sciences, 1, 30-40.

Wu, W., Tokuda, M., Kashiwagi, A., Henmi, A., Okada, Y., Tachibana, S., \& Nomura, M. (2015). Evaluation the fatty acid composition of the seeds of Mangifera indica L. and their application. Journal of Oleo Science, 64, 479-484. http://doi.org/10.5650/jos.ess 14238

Zheng, L. H., Huang, X., Wang, L., \& Chen, Z. X. (2012). Physicochemical properties, chemical composition and antioxidant activity of Dalbergia odorifera T. Chen seed oil. Journal of the American Oil Chemists Society, 89, 883-890. https://doi.org/10.1007/s11746-011-1967-9 


\section{Copyrights}

Copyright for this article is retained by the author(s), with first publication rights granted to the journal.

This is an open-access article distributed under the terms and conditions of the Creative Commons Attribution license (http://creativecommons.org/licenses/by/4.0/). 